\title{
A Random Model of Solid Waste Mixing on the Acting Grate
}

\author{
LI Xiang ${ }^{1,2, a}$, TANG Yike ${ }^{1, b}$, LIU Shuang ${ }^{2}$, YANG Lu ${ }^{2}$ \\ ${ }^{1}$ College of Mechanical Engineer Chongqing University, Chongqing, 400044, China; \\ ${ }^{2}$ School of Mechanical and Power Engineering Chongqing University of Science \& Technology, \\ Chongqing, 401331, China; \\ aE-mail: Ixcqust@outlook.com; bE-mail: stan1921@sina.com;
}

Keywords: acting grate, waste mixing, stochastic method, Markov chain

\begin{abstract}
The random walk model is used to describe the mixing of municipal solid waste on the acting grate. An experimental system has been developed for studying the vertical mixing of the waste on the grate. The tracer particles are used to study the mixing process, which are made according to the physical and geometrical properties of the waste that is collected directly in a region. The results show that there are close agreement between the random walk model and the experimental data. The random walk model can effectively describe the mixing process of the waste.
\end{abstract}

\section{Introduction}

The waste-to-energy combustion not only to significantly reduce the volume of waste, but also can recover energy from the combustible materials in the waste. In order to fulfill the increasingly stringent environment protection regulation, we do not just simply add the gas cleaning systems in the waste-to-energy system, and to improve the incineration efficiency of the grate is also an important means for controlling the emission of the pollutant.

The components of the municipal solid waste are diversiform and inconstant, so the physical and chemical properties of MSW are not accurately and reliably determined as other fossil fuels, it is one of the reasons for the low efficiency of the waste incinerator, so there have been many different types of the grates and the combustion chambers. [1] But at present there aren't the satisfactory MSW combustion models on the acting grate in the public documents, [2-4] to improve the incinerator is difficult due to the lack of reliable data and basic design process, these basic data also is used to assist incinerator operation and control.

The waste with the component heterogeneous is distributed randomly in the waste bed, and the waste particles contact each other (shown in Fig. 1). The waste particles are moved and mixed on the grate due to the force of gravity and the activities of reciprocating grate arising. The degree of the waste mixing is key factor for MSW incineration efficiency in the chamber. The importance and role of the mixing model has been recognized by

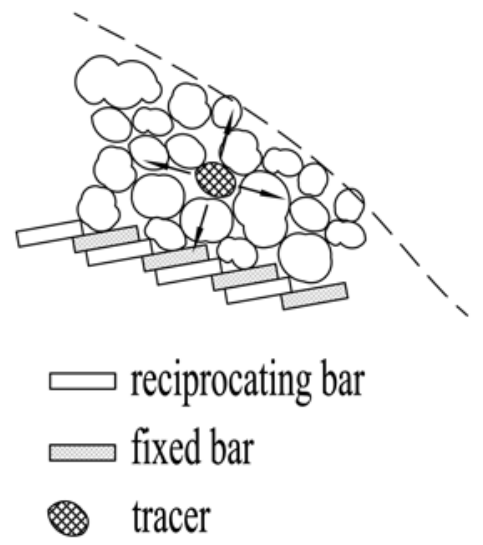

Fig. 1. Particle movement by reciprocating and fixed bar several researchers, and they also believe that efficiency of the existing grate system is not high. [5-8] The purpose of this study is to describe and quantify the mixing process in order to develop a mathematical model of the waste mixing. In this study, many tracer experiments have been taken in the full-scale experiment. The mixing model has been developed based on exchange position between adjacent particles in this paper, and the advantage of this model can test the different mixing techniques without the need for expensive pilot system. Currently, it has been proved that this mixing model is able to quantify the mixing of MSW in the vertical direction on the grate.

\section{Mathematical Model}

There are three principal mechanisms about the solid particles mixing: diffusion - the distribution of particles by the random motion of particles relative to other particles; convection transfer between adjacent particles by location - exchange in a mixture; shear - the change in the configuration of components by slip planes in mixture. All of these solid mixing mechanisms act on 
the mixing process of MSW on the moving grate with different degrees, but it is very difficult to research into the effect of each mechanism about the waste mixing on the grate. The convection mixing mechanism is dominant for interaction between the waste particles in the bed, and this interaction between the waste particles is taken by disturbance of the reciprocating of the grate bar. The shear mixing is taken place due to the different moving speed of waste which is on moving grate bar or fixed grate bar, so the shear mixing mechanism may not play a leading role as the fluid through a mixer.

The random walk model of Markov chain is used to study the waste mixing in this paper. Compared with the continuous model, because that MSW has some characteristics of particle, the discrete model is more suitable for simulation of waste mixing. Each mixing step is considered as one event in a discrete space-time at the stochastic model, so the model can be easily developed for solid mixing process. Because a large number of discrete particles are effected by any force or strain, it is an effective method to study the mixing process based on statistical method, especially Markov chain model. In fact, the Markov chain has been used to establish the solid particle mixing model by a number of scholars. [9-11]

The random walk problem is a Markov chain $\left\{X_{m}, m=1,2 \ldots\right\}$ in an integer dimensional state space, in this model, if the system is in a given state $k$, then system will be either remained state $k$ or transferred to the one of states that adjacent to state $k$ after one-step transition; in other cases, the system can only be transferred to the nearest neighbor. The transition probability matrix $P$ of the general random walk problem in the finite state can be expressed as follow (Eq.1):

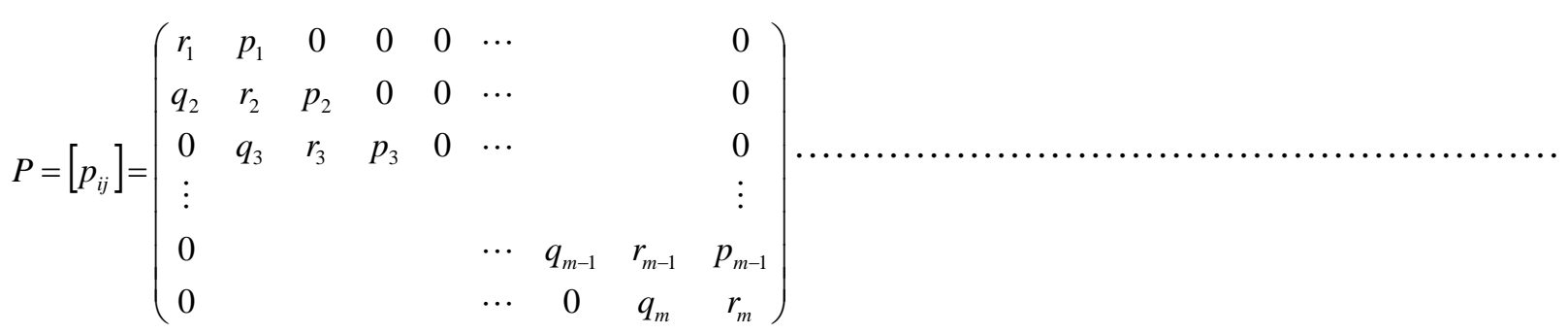

The $p_{k}, r_{k}$ and $q_{k}(k=1,2, \cdots, m)$ that are the non-negative real number in Eq. 1 can be expressed as follow (Eq.2):

$r_{l}+p_{l}=1$

$q_{k}+r_{k}+p_{k}=1, k=2,3, \cdots, m-1 ;$

$r_{m}+p_{m}=1$

This model can be used to simulate the physical processes including particle diffusion.

If $r_{l}=1, p_{l}=0$ and $r_{m}=1, q_{m}=0$, then the model is called the random walk model with absorption wall at $l$ and $m$ in Eq.1.

If $r_{l}>1, p_{l}>0$ and $r_{m}>1, q_{m}>0$, then the model is called the random walk model with reflective wall at $l$ and $m$ in Eq.1.

Because the mixing in the vertical direction on the grate significantly effect the incineration efficiency in the incinerator and it is ease to descript the problem in this paper, the model of the mixedness is established in this study in this direction. The experimental scheme is shown in Figure 2. The waste is mixed under turbulence of the acting grate and gravity, the bed is divided into $m$ layers along the vertical direction, and these layers can be considered as the states of the random walk. After each step for random walk, one waste particle can be either remained in the same layer or moved upward or downward to one layer (step). The waste particles are rarely moved more than

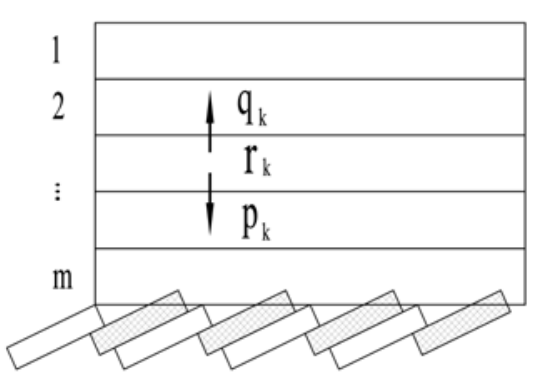

Fig.2. Cells of MSW Bed and the flow of particle one layer after each disturbance. The particle that is used to observe the position is called tracer after each step. The mathematical distribution of tracer relate to the initial distribution and transition probability matrix of the tracer after $n$ steps. It can be expressed as follow (Eq.3):

$c_{j}(n)=\sum_{i=1}^{m} c_{i}(0) p_{i j}^{n} \quad(j=1,2, \cdots, m)$

The vector $c_{j}(n)$ is the probability that the tracer is in the state $j$ after $n$ steps; the vector $c_{i}(n)$ is the initial probability of tracer; the $p_{i j}$ is the probability that the tracer transits from state $i$ to 
state $j$.The Eq.3 can be expressed in matrix form as follow (Eq.3a):

$c(n)=c(0) P^{n}$

If the tracer is located on the layer $i$ before mixing, the $c(n)=(0, \cdots, 0,1,0 \cdots 0)$, the final distribution of tracer can be calculated based on Eq.3 after any step with the random walk. If the initial vector and the transition probability matrix are given, the random walk can be fully determined. In order to determine the fluctuations in the layers of tracer after mixings, the variance can be calculated by the following equation:

$$
\begin{aligned}
& \sigma^{2}(n)=\frac{1}{m} \sum_{j=1}^{m}\left[c_{j}(n)-\frac{1}{m}\right]^{2} \\
& \sigma_{0}^{2}=\frac{1}{m}\left(1-\frac{1}{m}\right) \ldots \ldots \ldots . . . \\
& M=1-\frac{\sum_{j=1}^{m}\left[c_{j}(n)-\frac{1}{m}\right]^{2}}{1-\frac{1}{m}} \ldots . .
\end{aligned}
$$

Eq.6 expressed the degree of mixing after $n$ times mixing.

It is an important special case that upward probability and downward probability are equal in repeated independent step. For this example:

$$
\begin{aligned}
& p_{1}=p, r_{1}=1-p \\
& p_{k}=p, q_{k}=p, r_{k}=1-2 p ; k=2,3, \cdots, m-1 \\
& q_{m}=p, r_{m}=1-p
\end{aligned}
$$

So Eq.6 can be expressed as follow (Eq.7):

$$
P=\left(\begin{array}{ccccccc}
1-p & p & 0 & 0 & 0 & \cdots & 0 \\
p & 1-2 p & p & 0 & 0 & \cdots & 0 \\
0 & p & 1-2 p & p & 0 & \cdots & 0 \\
\vdots & & & & & \vdots & \vdots \\
0 & & \cdots & 0 & p & 1-2 p & p \\
0 & & \cdots & 0 & 0 & p & 1-p
\end{array}\right)
$$

Eq.7 is a double stochastic process, i.e. that sum of element of each column is 1 , then the equation has a steady-state distribution, it can be expressed as follow (Eq. 8):

$$
\left(\frac{1}{m}, \frac{1}{m}, \cdots, \frac{1}{m}\right)
$$

This means that a limit probability exists, i.e., the whole process will be in a steady state after a large number of transitions, which value is independent of the initial state. In the other words, the non-separated material will eventually reach a homogeneous state, which has a uniform degree of mixing, and it is independent of the initial position of the tracer. Theoretically, if the probability $p$ and $q$ can be found, not only the key will be resolved in waste mixing, but also the experimental data will be able to be fit best.

\section{Experiment}

\subsection{Instrument}

Because of restrictions by the size of the test apparatus, the untreated waste can not be used in the reduced scale model. On the other hand, due to difficulties in manufacturing, a full-size model is rarely used to study the mixing of MSW. But the full-size model can be used to study the bed with waste which is collected from the local community. This is an important consideration, because the directly collect waste can represent the similar geometric characteristics of waste used in 
incineration plant and the actual conditions of incinerator. Because of this, a full-scale model of forward acting grate is built in this paper, and directly collect waste mixing tests are carried out in the model.

The long of full-scale model used in this paper is $6 \mathrm{~m}$, height is $0.9 \mathrm{~m}$, width is $0.5 \mathrm{~m}$, and the forward acting grate is inclined 10 degrees to the horizontal. The bed is divided into five layers along the vertical direction, the thickness of each layer is $15 \mathrm{~cm}$. The height of grate bar is $13 \mathrm{~cm}$, the angle of bar between the bed is 45 degrees. The stroke of the reciprocating bar that moves from the bottom to the top is $50 \mathrm{~cm}$. The track of tracer is recorded by two digital cameras which are mounted on both sides of the apparatus. The motion probability of the waste particle in the bed can be determined in a horizontal direction and a vertical direction by this apparatus.

The size and density of tracer are obtained based on statistical data of the waste composition in the local region, i.e. the size and density of tracer are the average values of the waste. The tracer is made of foam rubber, which size is $12 \mathrm{~cm}$, and density is $221 \mathrm{~kg} / \mathrm{m}^{3}$.

\subsection{Experimental Procedure}

The waste used in the test is collected from the local region. The waste is filled in the apparatus, and the tracer is placed in the different layers of inlet. The moving frequency of reciprocating bar is 10 times per minute, and stroke is $500 \mathrm{~mm}$. After each reciprocating, the position of the tracer is recorded by two cameras. So after $n$ times reciprocation, the tracer can be tracked that moved from the initial position to the current position.

\subsection{Analysis of Data}

The position of tracer in the bed has be recorded after every reciprocation until the tracer particles

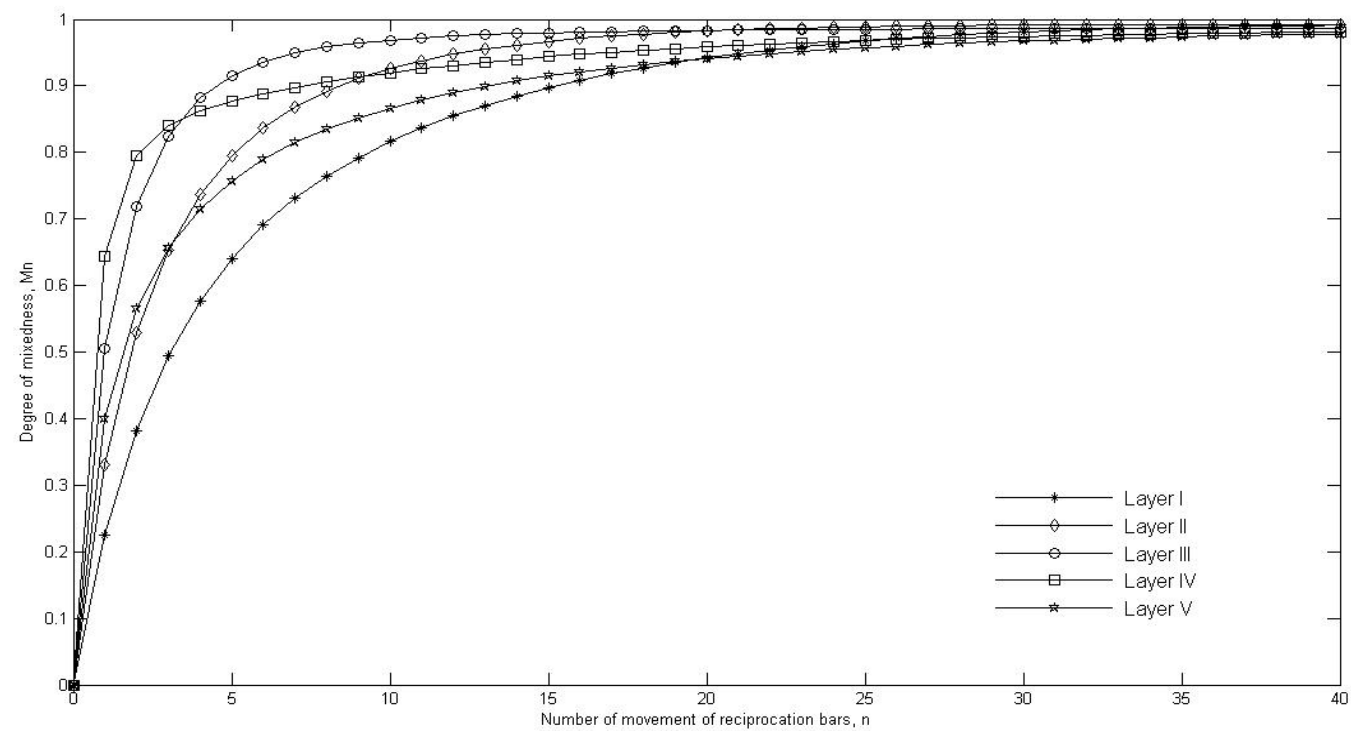

Fig.3. Degree of mixedness vs. number of passes for mixing wheat on the acting grate.

moved into the outlet after the $n$ times reciprocation. The residence count of the tracer in each layer of 5 layers is normalized. The degree of mixing $M$ can be calculated with Eq.6 in the vertical direction of bed, the results are shown in Table 1. Figure 3 shows the relationship between the degree of mixing and reciprocating times of the moving bar.

Table 1 Test Data Calculation \& Walk Model Simulation

\begin{tabular}{|c|c|c|c|c|c|c|c|c|c|c|c|c|c|c|c|}
\hline Step $n$ & 1 & 2 & 3 & 4 & 5 & 6 & 7 & 8 & 9 & 10 & 15 & 20 & 25 & 30 & 35 \\
\hline $\begin{array}{c}\text { Test data } \\
\text { calculation }\end{array}$ & 0.5283 & 0.6531 & 0.8625 & 0.8972 & 0.9055 & 0.9129 & 0.9257 & 0.9669 & 0.9743 & 0.9587 & 0.9682 & 0.9811 & 0.9890 & 0.9913 & 0.9918 \\
\hline $\begin{array}{c}\text { Walk } \\
\text { model } \\
\text { simulation }\end{array}$ & 0.5062 & 0.7192 & 0.8245 & 0.8820 & 0.9155 & 0.9361 & 0.9494 & 0.9582 & 0.9644 & 0.9689 & 0.9796 & 0.9833 & 0.9850 & 0.9859 & 0.9865 \\
\hline
\end{tabular}

\section{Results and Discussion}

Table 1 is a comparison of experimental observations and simulation calculations, the simulation 
data is calculated based on the Eq.6. Obviously, the random walk model can adequately describe the waste mixing process.

The waste mixing process can be observed from Fig.3. As expected, the degree of dispersion of waste increases with the number of the grate movement increasing. The mixing effect of waste in the upper and lower is poor, because that the waste in these two layers transfer only downward or upward, and the waste in upper part of the bed will easily roll down along the surface of the bed. The mixing effect of waste in the intermediate layer is preferable, because that the waste in the intermediate layer can move upward or downward by disturbance of the reciprocating.

\section{Conclusions}

The waste mixing can be described by the random walk model in the vertical direction. The test data and the prediction of the random walk model are very consistent. This model can be used to the waste combustion control and assist the grate mechanism design. If applying this method to the longitudinal direction and transverse direction, the model can also be extended to three-dimensional model to study the mixing in the vertical direction, parallel direction and the horizontal direction, i.e. the mixing and the moving path of the waste in the whole bed.

\section{Acknowledgement}

This research is funded by National Natural Science Foundation of China (Project No. 51305474), Chongqing Natural Science Foundation (Project No. cstc2013jcyjA70004) and Research Foundation of Chongqing University of Science \& Technology (Project No. CK2011B20).

\section{References}

[1] S. Wirtz, F. Krull, Modelling of waste grate firing for a complete furnace simulation, Proc. 1998 Int. Conf. Incineration Therm. Treat. Technol. (1998).287-291.

[2] V. Nasserzadeh, C. Schofield, D.W. Scott, A. Loader, A. Leonard, J. Swithenbank, Design optimization of Coventry municipal solid waste incinerator, J. Environ. Eng. 120(6), (1994) 1615-1629.

[3] M. Beckmann, C. Wiese, M. Davidovic, Gasification of waste materials in grate systems, Proc. 1997 Int. Conf. Incineration Therm. Treat. Technol. (1997)145-152.

[4] J. Buekens, A simulation model for a MSW grate incinerator, Solid Waste Management: Thermal Treatment and Waste-to-Energy Technologies, Conference of an International Specially Conference, 1995, 474-480.

[5] J.R. Holmes, Refuse Recycling and Recovery, 1981, 41-49, Chap. 3.

[6] N.H. Johnson, D.C. Reschly, MSW and RDF - an examination of the combustion process, Joint ASMErIEEE Power Generation Conference, 1986.

[7] A.M. Pelling, Municipal Waste Incineration, One Day Symposium on Managing Wastes in the 1990s, Institute of Waste Management, 1991.

[8] K. Whiting, Large Scale MSW Incineration Technologies, Conference on Incineration of Municipal Waste with Energy Recovery, 1997.

[9] M. Aoun-Habbache, An experimental method and a Markov chain model to describe axial and radial mixing in a hoop mixer, Powder Technology 128 (2002) 159-167.

[10] Nakamura, M., and N. J. Themelis, "Modeling of Solid Waste Flow and Mixing on the Traveling Grate of Waste- to-energy Combustion Chambers," Proc. 12th annual North American Waste To Energy Conference (NAWTEC12), Georgia. (2004).

[11] Ruey H. Wang and L. T. Fan, Axial Mixing of Grains in a Motionless Sulzer (Koch) Mixer, Ind. Eng. Chem., Process Des. Dev., 15(3), (1976) 381-388 